\title{
Obstructive sleep apnoea and obesity
}

\author{
Tintinger GR, ${ }^{\text {a Pretorius } L},{ }^{\mathrm{b}}$ Labadarios $\mathbf{D}$ \\ aDepartment of Internal Medicine, Steve Biko Academic Hospital, Faculty of Health Sciences \\ University of Pretoria, Pretoria \\ ${ }^{b}$ Medical Research Council Unit for Inflammation and Immunity, Department of Immunology \\ Faculty of Health Sciences, University of Pretoria \\ cHuman Sciences Research Council, Population Health \\ Health Systems and Innovation (PHHSI), Cape Town \\ Correspondence to: Dr GR Tintinger, e-mail: grtintinger@gmail.com \\ Keywords: obstructive sleep apnoea and obesity
}

\section{Abstract}

The global obesity epidemic continues to gain momentum, and South Africa is one of those countries with an extremely high prevalence of obesity. The reported association between obstructive sleep apnoea (OSA) and obesity has resulted in a parallel increase in the incidence of OSA. Obesity and OSA interact by inducing systemic inflammation, metabolic aberrations and endocrine abnormalities, all of which predispose patients to atherosclerosis and cardiovascular disease. OSA is associated with a pro-inflammatory state, and increased serum C-reactive protein concentrations. Cardiovascular complications of OSA include myocardial infarction, stroke, congestive heart failure, hypertension and cardiac arrhythmias. The complications of OSA and obesity result in reduced quality of life, significant morbidity, and increased mortality, for untreated patients. OSA causes symptoms such as snoring, apnoea, excessive daytime sleepiness, and morning headaches, but $80-90 \%$ of patients are never diagnosed, or treated for their condition. Recognition of these symptoms, and referral of patients for a polysomnogram (home- or laboratory-based), expedites the diagnosis and therapy of OSA. While continuous positive airway pressure (CPAP) remains the mainstay of therapy, dietary measures are important, and should be implemented. Loss of weight can lead to significant reductions in the apnoea-hypopnoea index (AHI) of patients with OSA, and obese patients with OSA should aim for a $10 \%$ reduction in body weight.

\section{Introduction}

Modern society is currently faced with a serious global obesity epidemic, which appears particularly prevalent in the USA. ${ }^{1}$ However, obesity is also a major concern in South Africa. Prevalence rates for adult men and women, as high as $29 \%$ and $57 \%$ respectively, have been reported. ${ }^{2}$

Importantly, children are also becoming obese early in life. The obesity epidemic is associated with another serious, unrecognised epidemic due to obstructive sleep apnoea (OSA). There is a consistent relationship between obesity and OSA, with a body mass index $(\mathrm{BMI}) \geq 30 \mathrm{~kg} / \mathrm{m}^{2}$ having been reported in $60-90 \%$ of OSA patients. As the prevalence of obesity increases, there is likely to be a parallel increase in the prevalence of OSA. ${ }^{3}$ The prevalence of OSA in the adult population is estimated to be about $25 \%$, rising to $45 \%$ in obese individuals. ${ }^{3}$ OSA is more common in men and the elderly, but can also occur in children and young adults.

Despite the fact that 1 in 4 Americans are considered at high risk for OSA, only $10-20 \%$ of patients in the USA are diagnosed with the condition. ${ }^{4}$ Worldwide, estimates are far worse, with only one per cent of patients with OSA receiving appropriate therapy for their disease.
This review will discuss the importance and pathogenesis of OSA, describe the links between obesity, inflammation, the metabolic syndrome and OSA, as well as focus on diagnostic strategies and therapy, including diet and nutrition.

\section{Importance of OSA}

OSA is important due to its increasing prevalence worldwide. Despite being so common, over $80 \%$ of individuals with moderateto-severe disease are never diagnosed. A recent study among patients with type 2 diabetes in the USA, documented an $86 \%$ prevalence of undiagnosed $0 \mathrm{SA} .{ }^{5}$ The latter is thought to result in the progression of OSA and its related complications. OSA is recognised as an independent risk factor for hypertension, stroke, myocardial infarction, arrhythmias, and cardiovascular events. ${ }^{6}$ Furthermore, OSA is often closely associated with other conditions which are recognised causes of morbidity and mortality such as obesity, metabolic syndrome, atherosclerosis, systemic inflammation, insulin resistance. and type 2 diabetes mellitus. ${ }^{7,8}$ It has been reported that untreated OSA may shorten the lifespan of patients by up to 20 years. $^{9}$

OSA also impact. adversely on the quality of life of patients due to the symptoms and complications of the disease. OSA may result in memory impairment, poor performance at work and an increased risk of motor vehicle accidents. ${ }^{10,11}$ 
Healthcare costs are increased significantly as a result of untreated OSA. and estimates from the USA report an additional annual cost of $\$ 3.4$ billion attributable to unrecognised and untreated OSA. ${ }^{12}$

\section{Pathogenesis}

Obstructive sleep apnoea occurs during sleep when the muscles of the upper airway relax, resulting in partial (hypopnea) or complete (apnoea) obstruction to airflow. This loss of muscle tone leads to a collapse of the upper airway during sleep, especially if a predisposing factor such as obesity is present. ${ }^{13}$ Apnoeas and hypopneas are especially pronounced in the supine position during REM (dream) sleep and result in arousals from sleep, and enhanced respiratory effort towards the end of the event. In addition, oxygen saturation often falls during, or just after, these events, and is restored when activation of the sympathetic system occurs in response to nocturnal hypoxia. ${ }^{14}$ As soon as airflow and oxygenation return to normal, sleep usually continues. Recurrent arousals and sympathetic stimulation are associated with poor quality sleep as the cycles of obstruction, followed by restoration of airflow at the termination of events, continues throughout the night, sometimes repeating a number of times per hour. The measured episodes of apneas, plus hypopneas, per hour, is used to quantify the severity of disease as the apnoeahypopnea index (AHI).
Importantly, during the sympathetic activation associated with apnoeas, a patient's blood pressure may reach extremely high levels, and many patients with OSA have loss of the normal physiological pattern of "nocturnal dipping". ${ }^{6}$ Systolic and diastolic blood pressure normally decreases during non-REM sleep to rest the cardiovascular system. Loss of nocturnal dipping in patients with OSA is associated with a poorer outcome and higher cardiovascular risk. ${ }^{15}$

The aetiology of intermittent upper airway obstruction during sleep can be multifactorial, and usually depends on the loss of pharyngeal muscle tone in a patient already at risk with a narrow upper airway, or crowded oropharynx..$^{13}$ Although most patients with OSA are obese, a significant number have an alternative predisposing factor, such as retrognathia, enlarged tonsils, hypothyroidism or a neurological condition.

\section{Link to obesity and the metabolic syndrome}

The link between obesity, OSA, and the metabolic syndrome has been extensively investigated. ${ }^{16}$ Obesity provides a common pathophysiological mechanism for both OSA and the metabolic syndrome. Indeed, many patients with OSA and obesity have evidence of glucose intolerance, dyslipidaemia, hypertension, systemic inflammation or central obesity, all of which are significant risk factors for atherosclerosis. ${ }^{17}$ OSA, even in patients without obesity, may cause hypertension, systemic inflammation, and insulin

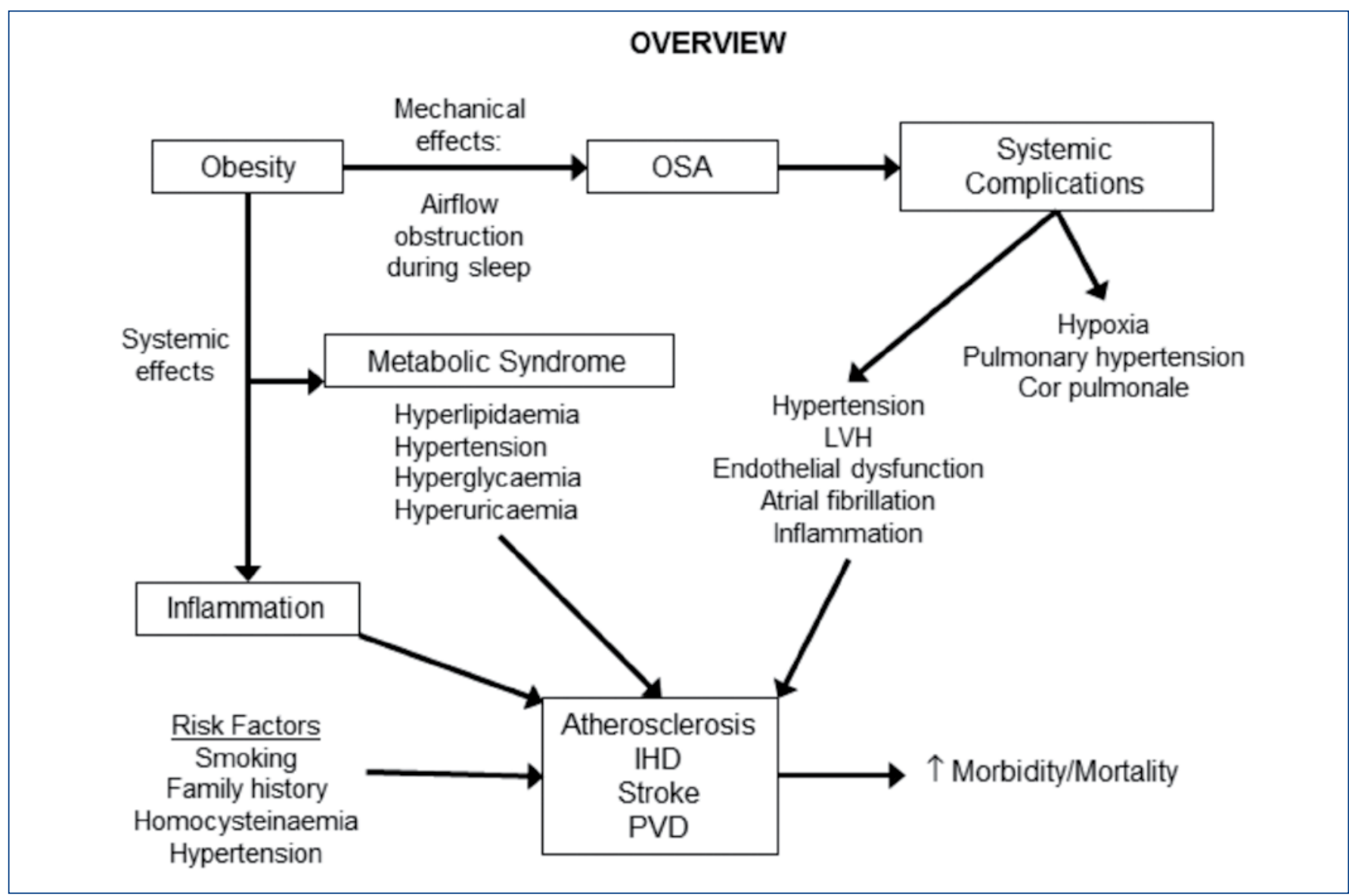

Figure 1: An overview of the interactions between obesity, obstructive sleep apnoea (OSA), metabolic syndrome, systemic inflammation and atherosclerosis

OSA is initiated by the mechanical effects of airflow obstruction, which result in hypoxia, inflammation, left ventricular hypertrophy and endothelial dysfunction. Obesity predisposes to both OSA, and the metabolic syndrome, and both of these conditions can lead to atherosclerosis, ischaemic heart disease, strokes, and peripheral vascular disease. Patients with additional risk factors such as smoking, or a family history of cardiovascular disease or hypertension, are at especially high risk of atherosclerosis and peripheral vascular disease, which increases morbidity and mortality. 
resistance. The high prevalence of OSA in patients with type 2 diabetes $(86 \%)$ renders these patients susceptible to complications from both diseases, and places them at high risk of atherosclerosis and cardiovascular disease. ${ }^{5}$ Diabetic patients with OSA may experience worsening of their glucose control, which improves when the OSA is appropriately treated..$^{18}$

An additional underlying factor which links obesity, OSA, and the metabolic syndrome, is systemic inflammation. Obesity is a systemic inflammatory disease, and is associated with low-grade systemic inflammation and endothelial dysfunction. Elevated levels of the inflammatory marker, C-reactive protein (CRP) have been reported in obese individuals. OSA itself further exacerbates systemic inflammation in obese patients. ${ }^{19}$ OSA initiates a cascade of events which, although initially mechanical in nature, results in the activation of macrophages, which release pro-inflammatory cytokines, namely tumour necrosis factor-alpha (TNF- $\alpha$ ) and interleukin-1 (IL-1), the so called endogenous mediators of inflammation. ${ }^{20}$ If the OSA remains untreated, the cycle of macrophage activation and release of proinflammatory cytokines continues, and a state of para-inflammation is established, a setting for ongoing endothelial dysfunction and progression of atherosclerosis (Figure 1). ${ }^{20,21}$

A number of markers of systemic inflammation are elevated in obese, and non-obese, patients with OSA, including TNF- $\alpha$, IL-6 and CRP. These markers tend to decrease with CPAP therapy, in both the obese and lean subjects, suggesting that OSA is at least partly responsible for the systemic inflammatory response.

Interestingly, leptin levels are also increased in patients with OSA, especially those with co-morbid obesity, but also in lean individuals. Leptin resistance in OSA has been linked to the effects of inflammatory cytokines. ${ }^{17}$ Leptin concentrations tend to decrease following therapy for OSA. The systemic nature of OSA is also responsible for many of the extra-pulmonary features of the disease resulting in multi-organ dysfunction, such as a procoagulant state, cardiac failure, atherosclerosis and proteinuria. ${ }^{19}$

\section{How to recognise OSA}

Patients with OSA may complain of symptoms that occur at night, such as excessive snoring, gasping or choking sensations during sleep, breathing pauses during sleep (witnessed apnoeas), nocturia, diaphoresis and poor quality sleep. Although $95 \%$ of patients with OSA complain of snoring at night, this symptom is not specific to OSA. ${ }^{11}$ Witnessed apnoeas have better diagnostic accuracy than snoring, but this symptom is not always present. ${ }^{11}$

Poor quality sleep, night after night, results in a wide spectrum of daytime symptoms. These include excessive daytime sleepiness, fatigue, morning headaches, memory impairment, an increased tendency to be involved in motor vehicle accidents, personality changes and depression, loss of libido, and poor concentration at work. ${ }^{11}$

Central obesity may be present in patients with OSA, and a waist circumference $>102 \mathrm{~cm}$ correlates with an increased AHI. A useful clinical screening tool is the STOP (snoring, tired, observed, pressure) Bang (BMl, age, neck circumference, gender) questionnaire (Table I), used by anaesthesiologists, which has a sensitivity of $92.9 \%$ and $100 \%$, for detecting moderate and severe OSA, respectively. ${ }^{22}$
Table I: The STOP Bang screening diagnostic tool

Symptoms, signs, predisposing factors Yes

1. Snoring: Do you snore loudly (loud enough to be heard through closed doors)?

2. Tired: Do you often feel tired, fatigued, or sleepy during the daytime?

3. Observed: Has anyone observed you stop breathing during your sleep?

4. Blood Pressure: Do you have, or are you being treated, for high blood pressure?

5. BMI: $\mathrm{BMI}>35 \mathrm{~kg} / \mathrm{m}^{2}$ ?

6. Age: Age $>50$ years old?

7. Neck circumference: neck circumference $>40 \mathrm{~cm}$ ?

8. Gender: male?

Interpretation: a positive answer ("Yes") to three, or more, of these symptoms, means the patient has a high risk of OSA.

Patients may also present with OSA complications, such as cardiac arrhythmias, including atrial fibrillation, difficult-to-control hypertension (drug-resistant hypertension), pulmonary hypertension, and congestive heart failure. ${ }^{7}$ OSA is highly prevalent among patients with cardiac failure, and may result in deterioration of cardiac function.

\section{Diagnosis}

A high index of suspicion is required by practitioners and dietitians or nutritionists to recognise the symptoms of OSA, so that patients can be referred for an appropriate diagnostic test. The diagnosis of OSA is confirmed by means of an overnight sleep study, or polysomnogram (PSG), which measures various parameters, including airflow, breathing activity and respiratory effort (Figure 2), oxygen concentration, brain EEG activity, leg muscle activity, and sleeping position.

The PSG can be performed at a sleep laboratory, or at the patient's home..$^{23} \mathrm{~A}$ recent study found that $76 \%$ of patients preferred to have their sleep study at home, and the available evidence indicates that the results of studies performed at home, or in a laboratory setting, for the diagnosis of OSA, are equivalent. ${ }^{23}$ The PSG is used to measure the $\mathrm{AHI}$, and to determine the level of CPAP necessary to treat the patient.

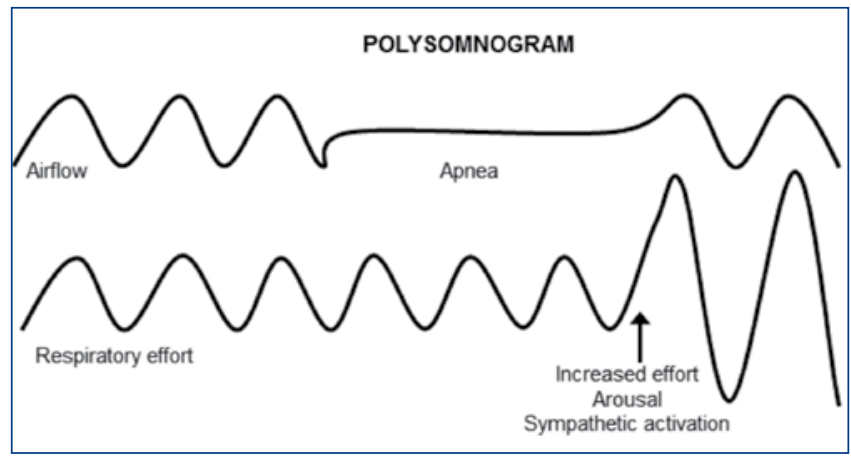

Figure 2: A schematic representation of a polysomnogram, showing cessation of airflow (apnoea) in the upper tracing, associated with an increased respiratory effort at the termination of the apnoeic episode in the lower tracing. This is usually associated with a sympathetic response, and a brief arousal from sleep. 
An AHI $\geq 5 /$ hour is diagnostic of OSA, and is considered mild in severity if $<15$ /hour. An index $\geq 15$ /hour indicates moderate OSA, while an $\mathrm{AHI} \geq 30$ /hour represents severe disease. This classification is important as it is used to guide subsequent therapy.

\section{Treatment}

CPAP is the effective treatment of choice for OSA. CPAP is effective, provided patients are able to tolerate the face mask and positive pressure. ${ }^{24}$ Most patients can select a well-fitting nasal mask, and often experience such a dramatic change in their quality of life, that they become long-term adherers to CPAP. Many patients sleep better, and are more awake during the day, able to concentrate, and insist on taking their machines whenever they go on holiday. However, some patients find it difficult to adapt to the wearing of the mask or the potove pressure, and despite all efforts they need an alternative form of therapy. This alternative form of treatment may include an oral appliance for mild OSA, or surgery for specific indications. A lifestyle intervention programme to lose weight can also have important benefits.

\section{Diet and OSA}

The high prevalence of obesity in patients with OSA, makes weight loss an attractive therapeutic option, and in theory at least, is a potentially reversible risk factor. A number of clinical trials have been conducted to evaluate the usefulness of dietary restriction in patients with OSA, and these have yielded mixed results. ${ }^{3}$ Some weight loss, even of a relatively small amount, can lead to a decrease in the patient's AHI. Although the relationship between the amount of weight lost, and the decline in AHI, is not linear, overall, it appears that for each kilogramme lost, the AHI decreases by $0.6-1$ / hour. ${ }^{25} \mathrm{~A}$ reasonable goal is a $10 \%$ decrease in body weight, which can result in a $26 \%$ change in the $\mathrm{AHI} .{ }^{26}$

Untreated OSA patients may have an increased appetite for refined carbohydrates, as well as increased ghrelin levels. ${ }^{3} \mathrm{~A}$ recent trial comparing patients treated with diet alone, CPAP plus diet, and an oral appliance plus diet, found that only the group on CPAP therapy was able to lose a significant quantity of weight. ${ }^{26}$ This suggests that CPAP therapy may be useful to treat OSA, and facilitate weight loss in these patients. ${ }^{26}$ However, not all studies have shown a beneficial effect of CPAP therapy. ${ }^{27}$

The effects of an intensive lifestyle intervention (ILI) in patients with type 2 diabetes with OSA, has also been studied, and compared to standard diabetes support and education (DSE). ${ }^{25}$ Especially during the first four months of the trial, patients in the ILI group, received portion-controlled diets with an energy content of 1200 $1500 \mathrm{kcal} /$ day for those $<113.6 \mathrm{~kg}$, and $1500-1800 \mathrm{kcal} /$ day for those patients weighing $\geq 113.6 \mathrm{~kg}$. The programme included 175 minutes/week of moderate intensity exercise activity (brisk walking). The DSE group participated in three sessions over a oneyear period, which included discussions on diet and exercise, as well as social support. Patients in the ILI group lost significantly more weight $(10.8 \mathrm{~kg})$, and their AHI decreased by a mean of $5 /$ hour (23/hour-18/hour), compared to the DSE group of patients, who did not lose weight, and whose AHI increased. This authors concluded that an intensive lifestyle intervention protocol can be effective in reducing the $\mathrm{AHI}$ among obese patients with type 2 diabetes. ${ }^{25}$

Although the decrease in $\mathrm{AHI}$ is usually small, this may be especially relevant for overweight patients with mild OSA (AHI 5-14/hour), who could potentially be cured if their OSA is due to obesity alone. The outlook for obese patients with moderate-to-severe OSA is less favourable, with only $5-10 \%$ of patients being able to sustain a significant weight loss beyond two years. ${ }^{3}$ However, even small changes in weight can have beneficial effects on blood pressure and blood glucose control..$^{28}$

\section{Conclusion}

OSA is a highly prevalent condition associated with obesity, and if untreated, is likely to result in serious complications. Effective therapy is available for OSA, which, when combined with measures such as dietary restriction, blood pressure control, and careful attention to reducing risk factors for atherosclerosis, can significantly improve the outlook for OSA patients.

\section{References}

1. Poirier P, Giles TD, Bray GA, et al. Obesity and cardiovascular disease: pathophysiology, evaluation and effect of weight loss. Circ. 2006;113(6):898-918.

. Van der Merwe M-T, Pepper MS. Obesity in South Africa. Obes Rev. 2006;7(4):315-322.

3. Romero-Corral A, Caples SM, Lopez-Jimenez F, Somers UK. Interactions between obesity and obstructive sleep apnea. Chest. 2010;137(3):711-719.

4. Hiestand DM, Britz P, Goldman M, Phillips B. Prevalence of symptoms and risk of sleep apnea in the US population. Chest. 2006;130(3):780-786.

5. Foster GD, Sanders MH, Millman R et al. Obstructive sleep apnea among obese patients with type 2 diabetes. Diabetes Care. 2009;32(6):1017-1019.

6. Selim B, Won C, Yaggi HK. Cardiovascular consequences of sleep apnea. Clin Chest Med. 2010;31(2):203-220.

7. Bradley TD, Floras JS. Obstructive sleep apnea and its cardiovascular consequences. Lancet 2009; 373: 82-93.

8. Malhotra A, Loscalzo J. Sleep and cardiovascular disease: an overview. Prog Cardiovas Dis 2009; 51 : 279-284.

9. Young T, Finn L. Epidemiological insights into the public health burden of sleep disordered breathing: sex differences in survival among sleep clinic patients. Thorax 1998; 53: 516-519.

10. Sateia MJ. Neuropsychological impairment and quality of life in obstructive sleep apnea. Clin Chest Med 2003; 24: 249-259.

11. MCNicholas WT. Diagnosis of obstructive sleep apnea in adults. Proc Am Thorac Soc 2008; 5: 154-160.

12. Kapur V, Blough DK, Sandblom RE et al. The medical cost of undiagnosed sleep apnea. Sleep 1999; 22: $749-755$.

13. Douglas NJ, Polo 0. Pathogenesis of obstructive sleep apnea/hypopnea syndrome. Lancet 1994; 344: 653-655.

14. Yaggi HK, Strohl KP. Adult obstructive sleep apnea/hypopnea syndrome: Definitions, risk factors and pathogenesis. Clin Chest Med 2010; 31: 179-186.

15. Del Colle S, Carra R, Rabbia F et al. Hypertension in obstructive sleep apnea. Vascular Disease Prevention 2005; 2: 29-35.

16. Vgontzas AN, Bixler EO, Chrousos GP. Sleep apnea is a manifestation of the metabolic syndrome. Sleep Med Rev 2005; 9: 221-224.

17. Arnaud C, Dematteis M, Pepin J-L, Baguet J-P, Lévy P. Obstructive sleep apnea, immune-inflammation and atherosclerosis. Semin Immunopathol 2009; 31: 113-125.

18. Lui MMS, Ip MSM. Disorders of glucose metabolism in sleep-disordered breathing. Clin Chest Med 2010; $31: 271-285$

19. Zamarron C, Paz VG, Riveiro A. Obstructive sleep apnea syndrome is a systemic disease. Current evidence. Eur J Int Med 2008; 19: 390-398.

20. Medzhitov R. Origin and physiological roles of inflammation. Nature 2008; 454: 428-435

21. Lavie L. Oxidative stress - A unifying paradigm in obstructive sleep apnea and comorbidities. Prog Cardiovasc Dis 2009; 51: 300-312.

22. Chung F, Elsaid H. Screening for obstructive sleep apnea before surgery: why is it important? Curr Opin Anaesthesiol 2009; 22: 405-411.

23. Skomro RP, Gjevre J, Reid J et al. Outcomes of home-based diagnosis and treatment of obstructive sleep apnea. Chest 2010; 138: 257-263.

24. Basner RC. Continuous positive airway pressure for obstructive sleep apnea. NEJM 2007; 356: 1751-1758.

25. Foster GD, Borradaile KE, Sanders MH et al. A randomized study on the effect of weight loss on obstructive sleep apnea among obese patients with type 2 diabetes. Arch Int Med 2009; 169: 1619-1626.

26. Lam B, Sam K, Mok WYK et al. Randomised study of three non-surgical treatments in mild to moderate obstructive sleep apnoea. Thorax 2007; 62: 354-359.

27. Kajaste S, Brander PE, Telakivi T et al. A cognitive-behavioral weight reduction program in the treatment of obstructive sleep apnea syndrome with or without initial nasal CPAP: a randomized study. Sleep Med 2004; 5: 125-131.

28. Tuomilehto HPI, Seppä JM, Partinen MM et al. Lifestyle intervention with weight reduction. First-line treatment in mild obstructive sleep apnea. Am J Respir Crit care Med 2009; 179: 320-327. 